\section{Correspondence Margarita Trobos margaritatrobos@gmail.com}

Received 18 September 2008 Revised 27 November 2008 Accepted 1 December 2008

\title{
Characterization of sulphonamide-resistant Escherichia coli using comparison of sul2 gene sequences and multilocus sequence typing
}

Margarita Trobos, ${ }^{1,2}$ Henrik Christensen, ${ }^{1}$ Marianne Sunde, ${ }^{3}$ Steen Nordentoft, ${ }^{4}$ Yvonne Agers $\varnothing,{ }^{5}$ Gunnar S. Simonsen, ${ }^{6,7}$ Anette M. Hammerum ${ }^{2}$ and John E. Olsen ${ }^{1}$

\footnotetext{
${ }^{1}$ Department of Disease Biology, Faculty of Life Sciences, University of Copenhagen, Grønnegårdsvej 15 st., 1870 Frederiksberg, Denmark

${ }^{2}$ National Center for Antimicrobials and Infection Control, Statens Serum Institut, Artillerivej 5, 2300 Copenhagen, Denmark

${ }^{3}$ National Veterinary Institute, Section of Bacteriology, Pb 750 Sentrum, 0106 Oslo, Norway

${ }^{4}$ National Veterinary Institute, Technical University of Denmark, Section for Poultry, Hangovej 2, 8200 Aarhus, Denmark

${ }^{5}$ National Food Institute, Technical University of Denmark, Bülowsvej 27, 1790 Copenhagen, Denmark

${ }^{6}$ Department of Microbiology and Infection Control, University Hospital of North Norway, Breivika, 9038 Tromsø, Norway

${ }^{7}$ Department of Microbiology and Virology, Faculty of Medicine, University of Troms $\emptyset$, Breivika 9038 Tromsø, Norway
}

The sul2 gene encodes sulphonamide resistance $\left(\mathrm{Sul}^{\mathrm{R}}\right)$ and is commonly found in Escherichia coli from different hosts. We typed $E$. coli isolates by multilocus sequence typing (MLST) and compared the results to sequence variation of sul2, in order to investigate the relation to host origin of pathogenic and commensal E. coli strains and to investigate whether transfer of sul2 into different genomic lineages has happened multiple times. Sixty-eight $E$. coli isolated in Denmark and Norway from different hosts and years were MLST typed and sul2 PCR products were sequenced and compared. PFGE was performed in a subset of isolates. All isolates were divided into 45 different sequence types (STs), with clonal complexes CC10, CC23, CC168, CC350 and CC69 being the most frequent. The sul2 gene from the majority of $E$. coli strains had only two point mutations, at positions 159 and 197, leading to a synonymous and a non-synonymous change, respectively. Five strains had extra single mutations. All poultry, poultry meat, and Danish human blood isolates had the same sul2 ST and some of these strains clustered under the same MLST STs, indicating that they shared habitats. Most PFGE profiles clustered according to source, but some included different sources. Sul ${ }^{R}$ E. coli from different animals, food, human faeces and infections did not cluster according to their origin, suggesting that these habitats share E. coli and sul2 gene types. However, while pig isolates on one occasion clustered with urinary tract infection isolates, poultry isolates seemed more related to isolates from bloodstream infections in humans. Presence of mainly two types of the sul 2 gene in both human and animal isolates, irrespective of date and geography, and the presence of both types in the same clonal lineages, suggest horizontal transfer of sul2.
Abbreviations: CC, clonal complex; DHPS, dihydropteroate synthase; MLST, multilocus sequence typing; ST, sequence type; UPGMA, unweighted pair group method using averages; UTI, urinary tract infection.

A supplementary figure and two supplementary tables are available with the online version of this paper.

\section{INTRODUCTION}

The known genes encoding plasmid-borne sulphonamide resistance $\left(\mathrm{Sul}^{\mathrm{R}}\right)$ are sul1, sul2 and sul3; each encodes a drug-resistant dihydropteroate synthase (DHPS) enzyme. Among them, the sul2 gene has previously been reported as 
the most prevalent one in Escherichia coli from pig and pork, poultry and poultry meat, cow and beef, mutton, human faeces and urinary tract infections (UTIs) (Blahna et al., 2006; Gow et al., 2008; Grape et al., 2003; Hammerum et al., 2006; Kerrn et al., 2002; Sunde \& Norstrom, 2006; Trobos et al., 2008). Based on a few partial sequences, this gene seems highly conserved, whether it is carried on small nonconjugative or large conjugative resistance plasmids (Radstrom \& Swedberg, 1988; Sorum \& L'Abee-Lund, 2002). It has been suggested that horizontal gene transfer plays a much larger role than clonal expansion in the spread of trimethoprim-sulfamethoxazole resistance (Blahna et al., 2006), but there is a need to investigate this further.

Multilocus sequence typing (MLST) is used to characterize E. coli isolates, among other bacteria, on the basis of sequence variation of seven housekeeping loci. The different nucleotide sequences of each locus are assigned different allele numbers, and then each strain is defined by the alleles of the seven loci (allelic profile). Closely related organisms can be grouped in clonal complexes. Analyses of E. coli isolates by MLST help to determine the genetic relatedness of the isolates, which is important for molecular epidemiological and evolutionary studies (Feil et al., 2004). MLST is used to study the population biology of microorganisms and provides an understanding of the population structure of important pathogens such as E. coli. Isolates that exhibit similar or identical MLST genotypes are very closely related and have descended from a recent common ancestor (Turner \& Feil, 2007).
In the present study, we applied MLST, followed by PFGE typing, to characterize sul2-positive strains of E. coli from different sources, and sequenced the sul 2 gene, with the following aims: (1) to see if the same clonal complexes contained resistant strains from different sources, which would be an indication that resistant strains are transferred between niches, and (2) to investigate whether horizontal gene transfer of the sul2 gene seems to have happened frequently.

Part of this study was presented at the 47th ICAAC, Chicago (USA), September 2007, and at the ASM Conference on Antimicrobial Resistance in Zoonotic Bacteria and Foodborne Pathogens, Copenhagen (Denmark), June 2008.

\section{METHODS}

Bacterial isolates. A total of 68 E. coli from different sources (pig, pork, poultry, poultry meat, mutton, healthy humans, blood stream infections and UTI), years (1969-2004) and countries (Denmark and Norway) were chosen for the study (Table 1). Except for the human blood isolates and the Norwegian animal isolates, the isolates were obtained from other studies (Hammerum et al., 2006; Kerrn et al., 2002; Sunde \& Norstrom, 2006; Trobos et al., 2008). They were selected by their resistance to sulphonamides and the presence of the sul2 gene. A breakpoint of $\geqslant 512 \mu \mathrm{g} \mathrm{ml}^{-1}$ was used for determining sulphamethoxazole resistance according to the Clinical Laboratory Standards Institute (CLSI).

MLST. We used the MLST protocol standardized for E. coli maintained at the Max Planck Institute for Infection Biology website (http://web.mpiib-berlin.mpg.de/mlst/dbs/Ecoli/documents/

Table 1. Variations in the sul2 gene of E. coli strains of animal, meat and human origin

\begin{tabular}{|c|c|c|c|c|c|c|}
\hline \multirow[t]{2}{*}{ Source } & \multirow[t]{2}{*}{ Year } & \multirow[t]{2}{*}{ Country } & \multirow[t]{2}{*}{ No. of strains } & \multicolumn{3}{|c|}{ No. of strains ${ }^{*}$} \\
\hline & & & & $\operatorname{sul} 2(\mathrm{a})$ & $\operatorname{sul} 2(\mathrm{~b})$ & sul2(c) \\
\hline \multicolumn{7}{|l|}{ Human } \\
\hline \multirow[t]{2}{*}{ Blood infections } & 1969 & Denmark & 6 & 6 & 0 & 0 \\
\hline & 2002 & Norway & 5 & 2 & 3 & 0 \\
\hline UTI & 2000 & Denmark & 5 & 2 & 3 & 0 \\
\hline Healthy (faeces) & 1997, 2002 & Denmark & 13 & 6 & 5 & 2 \\
\hline \multicolumn{7}{|l|}{ Pig } \\
\hline \multirow[t]{2}{*}{ Faeces } & 2002 & Norway & 5 & 3 & 2 & 0 \\
\hline & 2002,2003 & Denmark & 6 & $3 \dagger$ & 3 & 0 \\
\hline \multirow[t]{2}{*}{ Meat } & 2002 & Norway & 5 & $5 \ddagger$ & 0 & 0 \\
\hline & 2002,2003 & Denmark & 4 & 2 & 2 & 0 \\
\hline \multicolumn{7}{|l|}{ Poultry } \\
\hline \multirow[t]{2}{*}{ Faeces } & 2002 & Norway & 3 & 3 & 0 & 0 \\
\hline & 2004 & Denmark & 5 & 5 & 0 & 0 \\
\hline \multirow[t]{2}{*}{ Meat } & 2002 & Norway & 5 & 5 & 0 & 0 \\
\hline & 2004 & Denmark & 4 & $4 \S$ & 0 & 0 \\
\hline Mutton & 2001 & Norway & 2 & 1 & 1 & 0 \\
\hline
\end{tabular}

${ }^{\star}$ Number of strains belonging to one of the three sul2 gene variants [sul2(a), sul2(b) and sul2(c)]. Mutations are at positions 159, 197 and 427.

$\dagger$ One isolate contains an extra point mutation at position 672 ( $\mathrm{T}$ instead of $\mathrm{G}$ ).

¥One isolate contains an extra point mutation at position 288 (A instead of G).

$\S$ One isolate contains an extra point mutation at position 489 (A instead of T). 
primersColi_html). When two possible primers were given for the same direction, we chose the following: fumCR, gyrBR, purAF, recAF and $r e c A R$.

Sequencing of the PCR products was performed using the services of Macrogen. Sequences of the seven housekeeping genes ( $a d k, f u m C$, gyrB, icd, $m d h, \operatorname{pur} A, \operatorname{rec} A$ ) were analysed by BioNumerics using a special plug-in for MLST of E. coli.

PCR and sequencing of the sul2 gene. Presence of the sul2 gene was investigated by PCR using primers Sul2-F (5'-TTT CGG CAT CGT CAA CAT AA-3') and Sul2-R (5'-GTG TGT GCG GAT GAA GTC AG-3)' (primer concentration $10 \mu \mathrm{M}$ ). A multiplex PCR kit (Qiagen) was used with the following amplification conditions: heating for $15 \mathrm{~min}$ at $94{ }^{\circ} \mathrm{C}$, followed by 30 cycles at $94{ }^{\circ} \mathrm{C}$ for $30 \mathrm{~s}$, $48{ }^{\circ} \mathrm{C}$ for $90 \mathrm{~s}$ and $72{ }^{\circ} \mathrm{C}$ for $90 \mathrm{~s}$, followed by $72{ }^{\circ} \mathrm{C}$ for $10 \mathrm{~min}$. E. coli NCTC 50020 was used as positive control and E. coli DH5 $\alpha$ plasmid pUVP4401 was the negative control.

The sul2 PCR products (727 bp) were purified by using the NucleoFast 96 PCR plates (Macherey-Nagel) and sequenced at Macrogen. The sequences were analysed with KODON software (Applied-Maths) and compared by multiple alignment using CLUSTAL W and MEgA4 software (Tamura et al., 2007; Thompson et al., 1997).

PFGE. PFGE was used to type E. coli from poultry, poultry meat and Danish blood infections. The one-day PFGE Standardized Laboratory Protocol for Molecular Subtyping of E. coli O157:H7 from PulseNet (http://www.cdc.gov/pulsenet/protocols/ecoli_salmonella _shigella_protocols.pdf) was used with the following variation: the

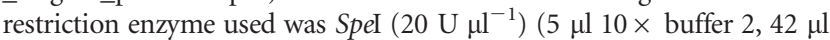
sterile water, $1 \mu \mathrm{l} \mathrm{BSA}$ and $2 \mu \mathrm{l} \mathrm{SpeI)}$. Digestions were performed in $50 \mu \mathrm{l}$ volumes.

Computer-assisted analysis of SpeI profiles was performed using the BioNumerics software (Applied Maths). The dendrogram was constructed using Dice similarity coefficients of SpeI PFGE patterns and the UPGMA clustering method (see Supplementary Fig. S1, available with the online version of this paper).

\section{RESULTS AND DISCUSSION}

\section{Diversity of sul2-positive E. coli STs among animals, food, humans and infections}

A minimal spanning tree $\left(\mathrm{MS}_{\mathrm{TREE}}\right)$ shows the distribution of the multilocus sequence types (STs) and their relation to the sul2 gene variants (see below) of the $68 \mathrm{E}$. coli isolates from all the sources (see Fig. 1).

Among the 68 E. coli isolates studied, 45 different STs were identified (Supplementary Table S1). The largest group of isolates $(24 \%)$ was part of the clonal complex ST10 (CC10). They originated from pigs $(n=2)$, pork $(n=2)$, poultry $(n=3)$, poultry meat $(n=2)$, healthy humans $(n=6)$ and blood infection $(n=1)$. This was not surprising, since CC10 is the biggest clonal complex of the species, and E. coli isolates from very different hosts are assigned to it (e.g. healthy human, UTI, septicaemia, diarrhoea, pig, dog, chicken and soil) (http://web.mpiib-berlin.mpg.de/mlst/ dbs/Ecoli/GetTableInfo_html). CC10 comprised mainly the non-pathogenic E. coli isolates of our study. One-third of the pig (3 out of 9) and pork (4 out of 11) isolates belonged to the clonal complex CC23. A UTI isolate was also associated with this group.

Seven isolates were part of CC168; they were isolated from poultry $(n=1)$, pig $(n=2)$, pork $(n=1)$, blood infections $(n=2)$ and UTI $(n=1)$. CC350 contained E. coli from poultry meat $(n=2)$ and blood infection $(n=1)$. Three isolates from poultry meat, a healthy human and a blood infection belonged to CC69. This clonal complex is mainly represented by human UTI and bacteraemia $E$. coli isolates, although it has also been found in cow and sheep isolates (Tartof et al., 2005).

Fig. 2 shows an eBURST diagram of the current E. coli MLST global population structure. The spatial distribution of Sul ${ }^{\mathrm{R}}$ E. coli in the population seemed random; however, we have not tested this statistically. The Sul ${ }^{\mathrm{R}}$ strains typed in this study were scattered among the rest of the Sul ${ }^{\mathrm{R}}$ and $\mathrm{Sul}^{\mathrm{S}}$ E. coli from all over the world. This indicates that there is not a cluster of this specific resistant population, and that all E. coli could potentially become $\mathrm{Sul}^{\mathrm{R}}$.

\section{Comparison of sul2 gene sequences}

In total six point mutations were recognized (Fig. 3). We decided to name these variants of the sul2 genes in the following manner: if a mutation resulted in an amino acid change in the DHPS protein we gave it a new letter. Extra non-synonymous mutations are simply named as the sul2 variant they derive from.

The first mutation, at position 159, was a silent thymine (T) or cytosine (C) third-codon substitution. The second, at position 197, was a $\mathrm{C}$ or adenine (A) second-codon nonsilent substitution leading to alanine or glutamic acid, respectively. Almost all of the E. coli strains tested fell into one of these two variants: sul2(a) (TC variant) or sul2(b) (CA variant) (GenBank accession numbers FJ200242 and FJ200243, respectively) (Table 1). Two isolates, within the TC variant, contained an extra non-synonymous mutation at position 427 (A instead of $G$ ); this mutation was named sul2(c) (GenBank accession number EU653286).

The sul2(a) variant was the most frequently found among the strains tested. Three strains with the sul2(a) variant contained an extra synonymous mutation at position 288, 489 or 672 (GenBank accession numbers EU653287, EU653288 and EU653289, respectively) (see Fig. 3).

This study confirms, as previously reported on a smaller sample (Radstrom \& Swedberg, 1988) that the sul2 gene is highly conserved, with few point mutations, though not identical as was previously concluded (Sorum \& L'AbeeLund, 2002). Presence of only two main types of the sul2 gene in both human and animal isolates, irrespective of time and geography, suggests horizontal transfer of the gene. It has been concluded by others that this had probably happened within a short time, since signs of more point mutations, indicative of genetic drift, were not found (Sørum \& L'Abee-Lund, 2002). However, there may also be 


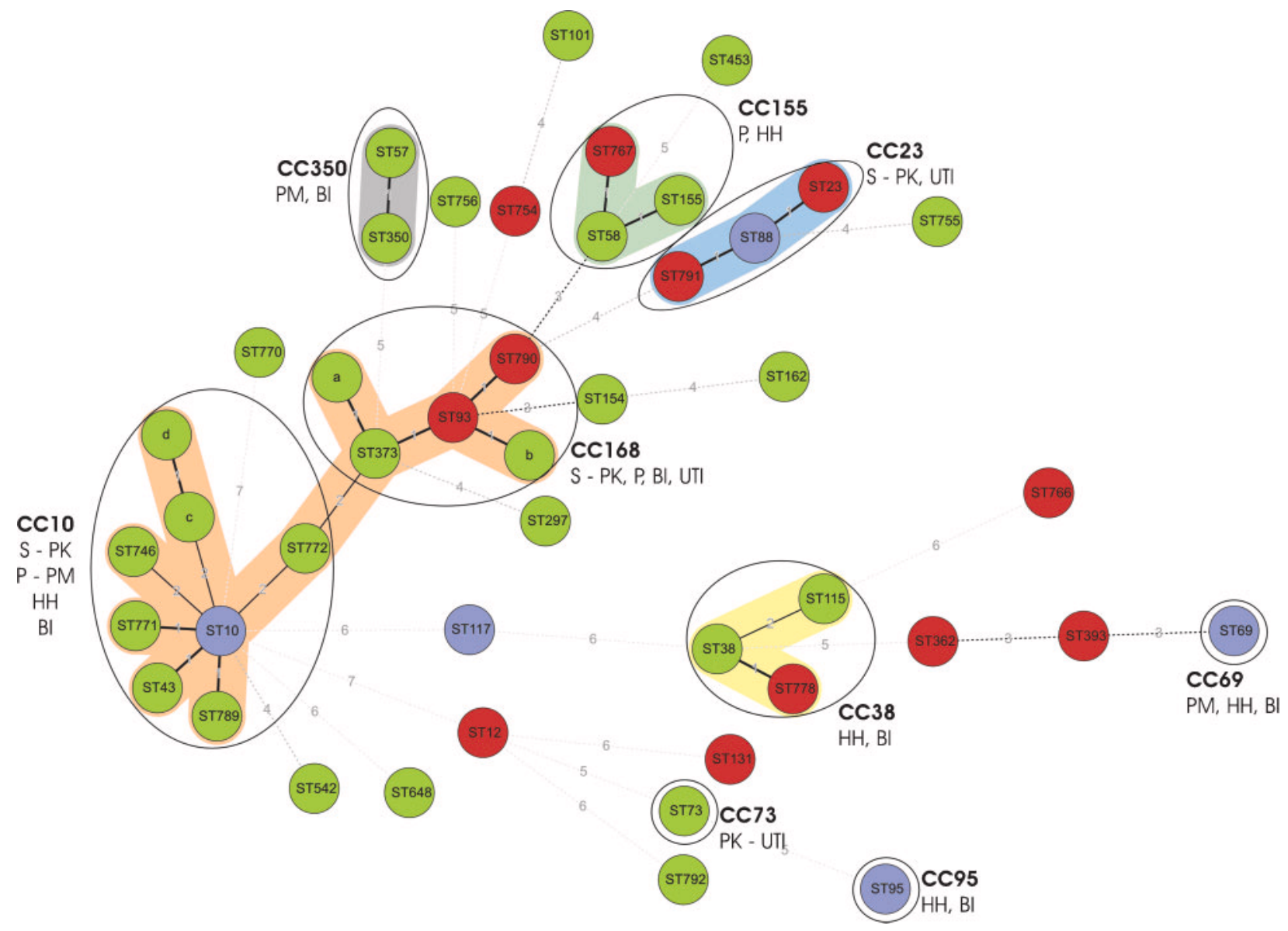

Fig. 1. Distribution of MLST STs and their relation to the sul2 gene variants within a minimal spanning tree (MS $\left.S_{\text {TREE }}\right)$ of 68 Escherichia coli from various sources. Each ST is represented by a circle. Ellipses containing several STs indicate clonal complexes (CC). The lines connecting the STs within a clonal complex are shaded in colours. Black lines connecting pairs of STs indicate that they differ in one allele (thick lines), two alleles (thin), or three to seven alleles (dotted). Green circles represent isolates that contain the sul2(a) gene variant, red circles represent the sul2(b) variant and blue circles represent both variants [except ST10, which contains the three variants: sul2(a), sul2(b) and sul2(c)]. S, swine; PK, pork; P, poultry; PM, poultry meat; $\mathrm{HH}$, healthy human; BI, blood infection; UTI, urinary tract infection.

a second possible explanation, namely in order to function efficiently as a DHPS enzyme, the protein cannot tolerate many changes to its amino acid sequence. Therefore, it could also have been that an ancient transfer occurred which resulted in a wide dissemination.

The two main sul2 STs were present in the same clonal lineage. This indicates that horizontal gene transfer has happened several times, into the same clonal lineage, i.e. spread of sul2 happens independently of the evolution of the clonal lineage.

\section{Link between poultry, poultry meat and human blood $E$. coli isolates and their sul2 gene ST}

Interestingly, all poultry, poultry meat, and Danish human blood isolates had the same sul2(a) variant and some of these strains clustered under the same multilocus STs (ST10, ST57 and ST69) (Supplementary Tables S1 and S2).
These isolates were PFGE typed in order to dismiss clonality (data not shown). We found that many PFGE profiles clustered ( $\geqslant 80 \%$ genomic similarity) (Supplementary Fig. S1). Most clustered according to their source, but there were exceptions to this (Supplementary Fig. S1). One cluster associated two poultry isolates, two isolates from poultry meat and one from a blood infection. Another linked one poultry strain with five human blood isolates. This is an indication that poultry isolates of E. coli may be a source of extra-intestinal $E$. coli infection in humans; however, investigations with more strains are needed to confirm this.

\section{Transfer mechanism of sul2}

Resistance genes can spread via two phenomena, horizontal gene transfer and clonal expansion. In this study, we found 


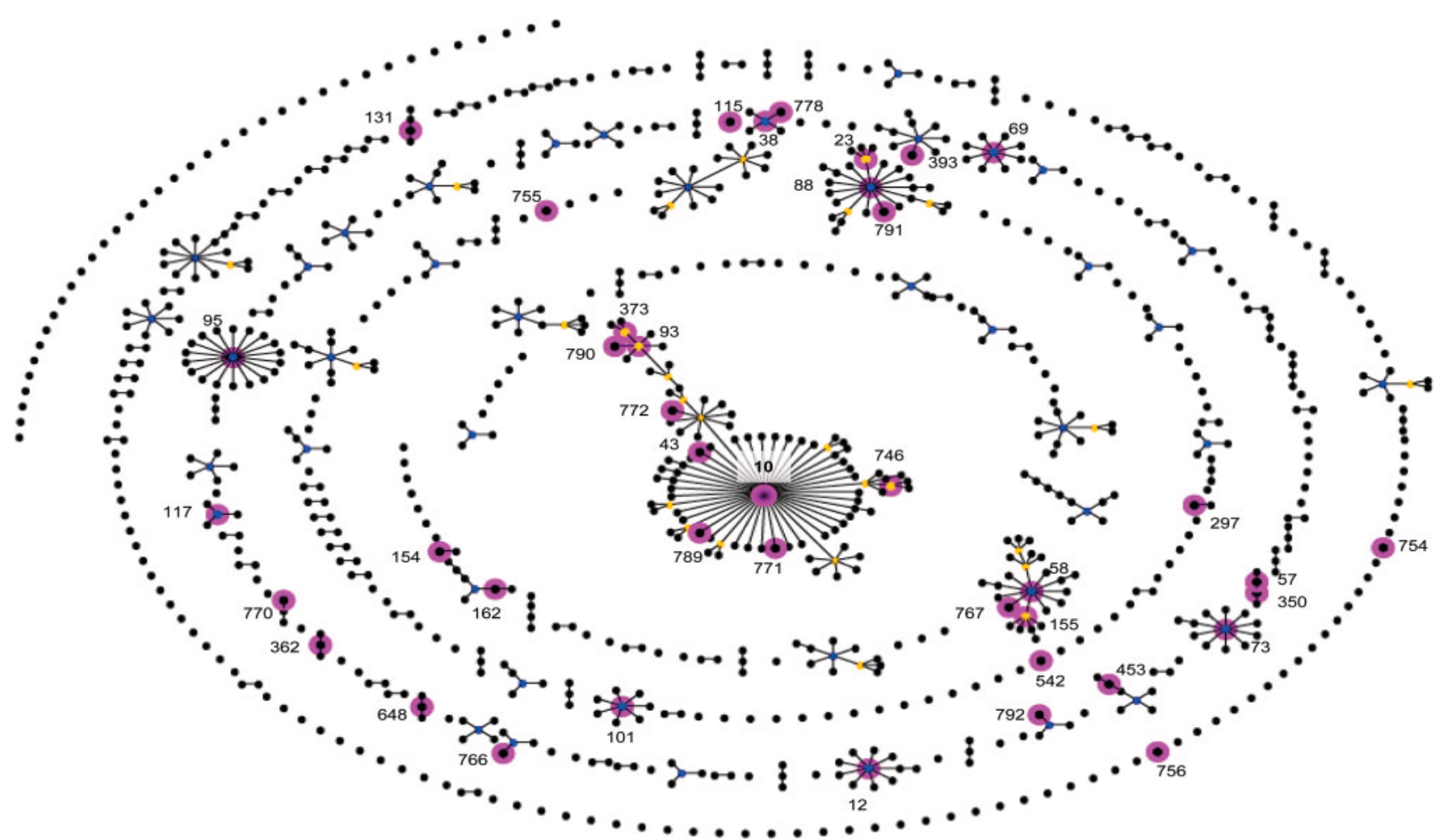

Fig. 2. Population snapshot of $E$. coli clusters of related and unrelated STs within the entire E. coli MLST database (performed using eBURST v3). This diagram compares the STs identified in this study (Sul ${ }^{\mathrm{R}}$ E. coli, with pink haloes) with all the E. coli isolates in the MLST database $\left(\mathrm{Sul}^{\mathrm{S}}\right.$ and $\mathrm{Sul}{ }^{\mathrm{R}}$ ). It does not show the genetic distance between unrelated STs and CCs. In each $\mathrm{CC}$ the primary founder is at a central position. A node between two STs represents a single-locus variation. [Performed on 11 September 2008.]

the same MLST STs, clonal complexes and sul2 sequence among E. coli isolates from very different ecological niches, regions and years, just as we found the same sul2 gene variant among different MLST types. In the first case, this suggests that strains were of clonal origin with a stable presence of sul2, while the other case indicates a widespread horizontal transfer of sul2 between different hosts. It may be that clonal lineages differ in their ability to act as recipients for horizontally transferred genes, in particular plasmid-encoded genes such as sul2.

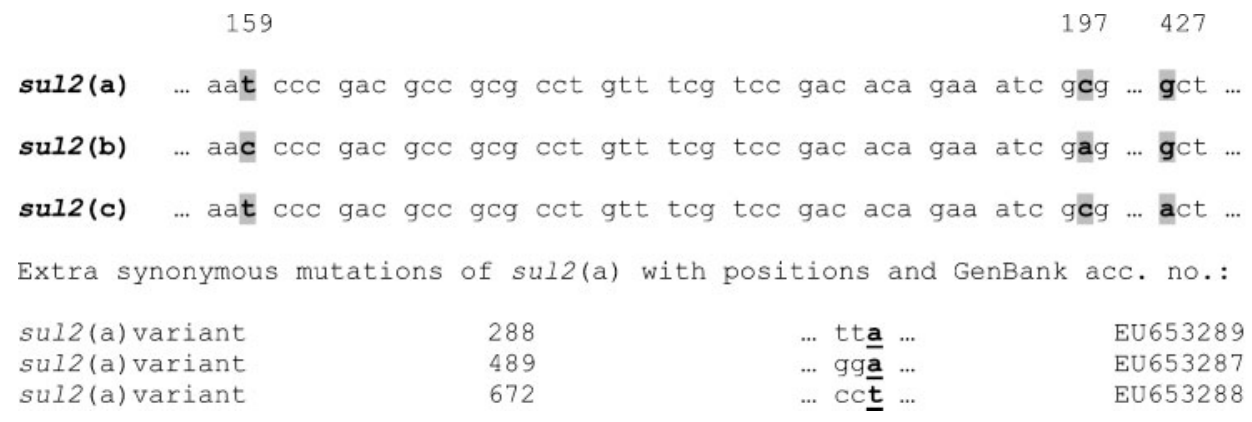

Fig. 3. Mutation sites in the sequence of the sul2 gene: representation of the three sul2 gene variants [sul2(a), sul2(b) and sul2(c)]. Mutations are at positions 159, 197 and 427. The extra mutations in three isolates derive from the sul2(a) type. These isolates contain an extra point mutation at position 288 (A instead of $G$ ), position 489 (A instead of $T$ ) and position 672 ( $T$ instead of $\mathrm{G}$ ), respectively. 
To conclude, this study shows that the same type of E. coli carrying an identical sul2 gene can colonize both animals and humans, and that strains which can be found among animals may be implicated in human infections such as UTI and septicaemia. Whether the strains or the sul2 gene transfer from animals to humans, or vice versa, cannot be assessed from the current study, due to the lack of a specific sul2 type colonizing each of the hosts.

\section{ACKNOWLEDGEMENTS}

Bacterial strains were provided by DANMAP (the Danish Integrated Antimicrobial Resistance and Research Program) and NORM/ NORM-VET (Usage of Antimicrobial Agents and Occurrence of Antimicrobial Resistance in Norway). This study is part of the multidisciplinary Early Stage Training (EST) site: TRAINAU 'Training Risk-Assessment In Non-human Antibiotic Usage'. It is funded by a European Marie Curie Host Fellowship for EST (MEST-CT-2004007819). The study was furthermore supported by a grant from the Scandinavian Society for Antimicrobial Chemotherapy.

\section{REFERENCES}

Blahna, M. T., Zalewski, C. A., Reuer, J., Kahlmeter, G., Foxman, B. \& Marrs, C. F. (2006). The role of horizontal gene transfer in the spread of trimethoprim-sulfamethoxazole resistance among uropathogenic Escherichia coli in Europe and Canada. J Antimicrob Chemother 57, 666-672.

Feil, E. J., Li, B. C., Aanensen, D. M., Hanage, W. P. \& Spratt, B. G. (2004). eBURST: inferring patterns of evolutionary descent among clusters of related bacterial genotypes from multilocus sequence typing data. J Bacteriol 186, 1518-1530.

Gow, S. P., Waldner, C. L., Harel, J. \& Boerlin, P. (2008). Associations between antimicrobial resistance genes in fecal generic Escherichia coli isolates in western Canadian cow-calf herds. Appl Environ Microbiol 74, 3658-3666.

Grape, M., Sundstrom, L. \& Kronvall, G. (2003). Sulphonamide resistance gene sul3 found in Escherichia coli isolates from human sources. J Antimicrob Chemother 52, 1022-1024.
Hammerum, A. M., Sandvang, D., Andersen, S. R., Seyfarth, A. M., Porsbo, L. J., Frimodt-Moller, N. \& Heuer, O. E. (2006). Detection of sul1, sul2 and sul3 in sulphonamide resistant Escherichia coli isolates obtained from healthy humans, pork and pigs in Denmark. Int J Food Microbiol 106, 235-237.

Kerrn, M. B., Klemmensen, T., Frimodt-Moller, N. \& Espersen, F. (2002). Susceptibility of Danish Escherichia coli strains isolated from urinary tract infections and bacteraemia, and distribution of sul genes conferring sulphonamide resistance. J Antimicrob Chemother 50, 513516.

Radstrom, P. \& Swedberg, G. (1988). RSF1010 and a conjugative plasmid contain sulII, one of two known genes for plasmid-borne sulfonamide resistance dihydropteroate synthase. Antimicrob Agents Chemother 32, 1684-1692.

Sorum, H. \& L'Abee-Lund, T. M. (2002). Antibiotic resistance in foodrelated bacteria - a result of interfering with the global web of bacterial genetics. Int J Food Microbiol 78, 43-56.

Sunde, M. \& Norstrom, M. (2006). The prevalence of, associations between and conjugal transfer of antibiotic resistance genes in Escherichia coli isolated from Norwegian meat and meat products. J Antimicrob Chemother 58, 741-747.

Tamura, K., Dudley, J., Nei, M. \& Kumar, S. (2007). MEGA4: Molecular Evolutionary Genetics Analysis (MEGA) software version 4.0. Mol Biol Evol 24, 1596-1599.

Tartof, S. Y., Solberg, O. D., Manges, A. R. \& Riley, L. W. (2005). Analysis of a uropathogenic Escherichia coli clonal group by multilocus sequence typing. J Clin Microbiol 43, 5860-5864.

Thompson, J. D., Gibson, T. J., Plewniak, F., Jeanmougin, F. \& Higgins, D. G. (1997). The CLUSTAL_X-Windows interface: flexible strategies for multiple sequence alignment aided by quality analysis tools. Nucleic Acids Res 25, 4876-4882.

Trobos, M., Jakobsen, L., Olsen, K. E., Frimodt-Moller, N., Hammerum, A. M., Pedersen, K., Agerso, Y., Porsbo, L. J. \& Olsen, J. E. (2008). Prevalence of sulphonamide resistance and class 1 integron genes in Escherichia coli isolates obtained from broilers, broiler meat, healthy humans and urinary infections in Denmark. Int J Antimicrob Agents 32, 367-369.

Turner, K. M. \& Feil, E. J. (2007). The secret life of the multilocus sequence type. Int J Antimicrob Agents 29, 129-135.

Edited by: S. D. Bentley 